\title{
Cantor's Proof in the Full Definable Universe
}

\author{
Laureano Luna
}

\author{
I. E. S. Doctor Francisco Marin \\ Philosophy, Siles, Spain \\ laureanoluna@yahoo.es \\ WILLIAM TAYLOR \\ Canterbury University \\ Mathematics and Statistics \\ Christchurch, New Zealand \\ w.taylor@math.canterbury.ac.nz
}

Received by Greg Restall

Published November 3, 2010

http://www . philosophy . unimelb.edu.au/ajl/2010

(C) 20 Io Laureano Luna and William Taylor

\begin{abstract}
Cantor's proof that the powerset of the set of all natural numbers is uncountable yields a version of Richard's paradox when restricted to the full definable universe, that is, to the universe containing all objects that can be defined not just in one formal language but by means of the full expressive power of natural language: this universe seems to be countable on one account and uncountable on another. We argue that the claim that definitional contexts impose restrictions on the scope of quantifiers reveals a natural way out.
\end{abstract}

KEYWORDS: Cantor's Theorem, constructive ordinals, countability, definability, indefinite extensibility, quantifiers, Richard's Paradox.

By the 'full definable universe' we mean to say the universe of all individual mathematical objects that are definable in natural language. It is often believed that each language has an at most countably infinite set of definitions because any natural language has a finite alphabet and admits only finitely long phrases. It seems to follow not only that in the full definable universe all sets are countable but that the universe has to be countable itself.

Since there seems to be no problem for the infinite set $\mathrm{N}$ of all natural numbers to exist in the full definable universe, it is natural to pose the question: what are we to do with Cantor's theorem in that universe? 
The answer seems fallaciously simple: in any definable universe, the powerset of $\mathrm{N}$ does not contain all the classically existing subsets of $\mathrm{N}$ but only the definable ones and these could make up only a countably infinite set. As well known, Richard's paradox stands in the way of such a solution. This paradox is most probably the cause that many definitionists prefer to frame their universes as models of this or that formal language: Richard's paradox is deemed to affect only languages for which the concept of definability is ill-defined, as seems to happen with natural languages. So the way out of the paradox appears to be the search for a formal language in which sufficiently many of mathematical objects can be defined.

But that way out has its own difficulty at least if the "sufficiently many" above is to include sets of sets of arbitrary large membership level: definability in any formal language L such that 'definable in L' is well-defined always falls short of definability simpliciter. To see this, take any such formal language $L$ and define the set $R_{L}$ of all sets definable in $L$ that are not self-membered. On the one hand, $R_{L}$ is well-defined for 'definable in L' is assumed to be well-defined; on the other hand, $R_{L}$ is obviously not definable in L. Surely, the full extent of 'definable' is the same as definable in the natural language. So, whenever we restrict ourselves to some formal language $L$ such that 'definable in L' is well-defined, we are mutilating the mathematical universe.

This is why we wish to address here the problem of Cantor's proof in the full definable universe, fully aware that addressing that problem is essentially the same as addressing Richard's paradox.

The following is a version of the Richard paradox. Take the set F of all English phrases that define sets of naturals; $F$ is countable; impose on $F$ a lengthalphabetical order OF and consider the enumeration EF of $F$ according to OF. Since EF is definable in English, the phrase 'the set containing exactly every natural number that is not a member of the set defined by the definition it is associated with in EF' is an English phrase defining a set of naturals; call it DF. DF is not in EF, contrary to the assumption that F contains all English phrases defining sets of naturals. So, there is no enumeration EF of all English phrases defining sets of natural numbers.

It is straightforward to render this argument an argument about definable sets of naturals instead of about definitions of such sets. Consider the set $S$ of all sets of natural numbers definable in English. It seems that $S$ has to be countable because the set $F$ of the corresponding definitions is countable. Eliminate from $F$ redundancies, keeping in each case the definition that occurs first in $E F$. This gives a definable enumeration ES of the definable sets of naturals and the enumeration gives a definable diagonal set DS of natural numbers that is not in ES. Hence there is no enumeration ES of all sets of naturals definable in English. 
Since the paradox relies essentially on the apparently obvious linguistic fact that the set of all English definitions of sets of natural numbers is countable, we shall hereafter focus on definitions of sets of naturals rather than on definable sets of naturals.

Note that $F$ has been defined above as a set of phrases, that is, as a set of strings of letters, a set of purely syntactic objects. It is obvious that the set $F$ is enumerable if it exists, as it is obvious that the enumerability of $F$ would lead to contradiction. Hence $F$ does not exist. There are only two reasons why $F$ could fail to be a set: either it is ill-defined or it is too large to be a set. Since $F$ would be enumerable (hence, presumably, not too large for sethood) if existing, $F$ must be ill-defined. Now there is a syntactical part to the definition of 'English phrase defining a set of natural numbers' and there is also a semantical component. Syntactical facts (such as being composed of a finite sequence of such and such symbols) are hardly ill-defined. So the ill-definedness must lie in the semantical side of the question; that is to say, the property of expressing in English a definition of a set of natural numbers must be ill-defined. Note that this is the property expected to separate $F$ from undoubtedly well-defined sets, as the set of all finite strings of letters from the Latin alphabet, for instance. This property will be called "P" here. So P is the property:

" $x$ expresses an English definition of a set of natural numbers"

The rest of this paper is mostly an attempt at clarifying in what sense $P$ and $F$ are ill-defined.

It should be acknowledged from the beginning that requiring from $\mathrm{F}$ to be decidable or effectively enumerable in order to be well-defined would be an extraordinary demand even for definitionists or constructivists, for there are lots of definable but not effectively enumerable collections most definitionists and constructivists usually take to be sets, for instance, the set of all computable functions. In spite of this, the claim that the set $\mathrm{E}$ of all precisely definable real numbers, though existent, is in some sense ill-defined because it is not effectively enumerable was early made by Borel [2]-so early in fact that the concept of effective enumerability had not yet be given any precise mathematical content - and proposed as a way out of the original Richard's paradox. Borel writes:

Mais l'ensemble E n'est pas effectivement énumérable, c'est à dire qu'on ne peut pas indiquer, au moyen d'un nombre fini de mots, un procédé sûr pour attribuer sans ambigüité un rang déterminé à chacun de ses éléments [...]. Telle est la réponse qu'on doit faire au paradox de $M$. Richard et à tous les paradoxes analogues: il est impossible de discuter effectivement sur un problème ou tous les termes ne sont pas explicitement définis.

[2] p. 446-7] 
That is to say:

But the set $E$ is not effectively enumerable, that is, it is not possible to specify, by means of a finite number of words, a secure procedure for attributing without ambiguity an order number to each of its members [...]. Such is the response that must be given to the paradox of M. Richard and all analogous ones: it is impossible to deal with a problem where not all terms are explicitly defined.

Now let us argue that the ill-definedness of $F$ revealed by the paradox is not a sort of fuzziness due to some kind of vagueness of the corresponding concept. Certainly, the concept of 'English definition of a set of natural numbers' is vague and has a fuzzy extension, for it is not clear-cut what should count as such a definition. Nevertheless, this fuzziness does not seem to account for the paradox because we can put forth a crisped version of it. We can use for the purpose Baaz's $\Delta$-operator [I], as follows.

Let $P(x)$ be the predicate ' $x$ expresses an English definition of a set of natural numbers' and $v$ a truth-value function with range in the interval $[0,1]$. That $\mathrm{P}(\mathrm{x})$ is fuzzy usually means that $v(\mathrm{P}(\mathrm{x}))$ can be other than 1 and other than 0 . The $\Delta$-operator works in the following way:

$$
\begin{aligned}
& \forall x(v(\mathrm{P}(\mathrm{x}))=1 \rightarrow v(\Delta \mathrm{P}(\mathrm{x}))=1) \\
& \forall x(v(\mathrm{P}(\mathrm{x})) \neq 1 \rightarrow v(\Delta \mathrm{P}(\mathrm{x}))=0)
\end{aligned}
$$

If we retain only those $x$ for which $v(\Delta P(x))=1$, we get the crisp core of $P(x)$, namely $\Delta \mathrm{P}(\mathrm{x})$. The predicate $\Delta \mathrm{P}(\mathrm{x})$ can be empty but hardly fuzzy, for suppose there is an $x$ such that $v(\Delta \mathrm{P}(\mathrm{x}))=\mathrm{r}$, with $0<\mathrm{r}<1$; then $v(\mathrm{P}(\mathrm{x})) \neq 1$; hence $v(\Delta \mathrm{P}(\mathrm{x}))=0$. This is why systems containing Baaz's operator usually include a necessitation rule that permits us to pass from $\Delta p$ to $\Delta \Delta p$.

We can read ' $\Delta \mathrm{P}$ ' as 'definitely-P'. As there seems to be little doubt of the existence of English phrases that are definitely English definitions of sets of naturals, we can reject the possibility that $\Delta \mathrm{P}(\mathrm{x})$ have an empty extension. Then we can revisit the paradox for the set $\Delta \mathrm{F}$ of English phrases that are definitely English definitions of sets of natural numbers, since any clearly defined length-alphabetical order of them will give us a definite diagonal definition $\Delta \mathrm{DF}$ of a set of naturals and $\Delta \mathrm{DF}$ will not be in $\Delta \mathrm{F}$.

Our point is that the ill-definedness of $\Delta \mathrm{P}$ and $\Delta \mathrm{F}$ is not primarily the fuzziness due to vagueness but the set theoretical kind of ill-definedness called 'indefinite extensibility'. The name is due to M. Dummett though the concept is due to Russell [I2] and can be said to have been implicitly suggested in 1906 by Poincaré $[\mathbf{I 2}]$ in the context of his discussion of Richard's antinomy. Dummett [4, 195-6] writes:

The reason why the ordinary concept of 'natural number' is inherently vague is that a central feature of it, which would be involved in any characterisation of the concept, is the validity of induction with respect to 
any well-defined property; and the concept of a well-defined property in turn exhibits a particular variety of inherent vagueness, namely indefinite extensibility. A concept is indefinitely extensible if, for any definite characterisation of it, there is a natural extension of this characterisation which yields a more inclusive concept; this extension will be made according to some general principle for generating such extensions, and, typically, the extended characterisation will be formulated by reference to the previous, unextended, characterisation.

A few comments on this passage are in order. First of all, it is not clear at all that the impossibility of characterizing the concept of natural number in first order languages can be taken as a proof of the 'inherent vagueness' of the concept.

Furthermore, Dummett should probably not have regarded indefinite extensibility as a form of vagueness. A concept $\mathrm{C}$ is vague whenever its extension $\hat{\mathrm{C}}$ is fuzzy, that is, $\hat{\mathrm{C}}$ has an imprecise contour surrounded by borderline cases $x$ such that $x \in \hat{C}$ has no classical truth value. Fuzziness is, so to say, a borizontal phenomenon. Indefinite extensibility affects not only vague concepts but non vague concepts as well (such as the concept of ordinal number) and is a vertical phenomenon: the extensions of indefinitely extensible concepts are not imprecisely distinguished from those of neighboring concepts; they are rather, so to say, topless, open ended.

In any case, the extensible aspect of an extensible concept is its extension, its intension remaining the same throughout. Indeed, a concept's intension bears the concept's identity; so, in order to say that a particular concept $\mathrm{C}$ is extensible, the intension of $\mathrm{C}$ must stay the same at all stages in the process of expansion of its extension. Hence, we must understand Dummett's words:

A concept is indefinitely extensible if, for any definite characterisation of it, there is a natural extension of this characterisation which yields a more inclusive concept

as stating that for any (purported) characterization of the extension of an indefinitely extensible concept, there is a more inclusive characterization of that extension.

Finally, the existence of a general procedure to produce such extensions, a procedure that employs reference to the previous characterization, can be taken as the existence of a diagonalization procedure.

Now, consider again our crisped version of Richard's paradox. Assume that whenever we define the set $\Delta \mathrm{F}$ of all English finite phrases that are definitely definitions of sets of naturals, we are defining a countable set (this is arguably so, see below, Section IV); then this set can never be taken as the ultimate extension of the corresponding crisped concept $\Delta C$, for there is always a diagonalization procedure that provides an object $\Delta \mathrm{DF}$ that is not in $\Delta \mathrm{F}$ though it falls under $\Delta \mathrm{C}$.

Therefore, we must admit that our 'all' in the definition is never the ultimate 'all' concerning finite English phrases that definitely count as definitions 
of sets of naturals. The scope of the quantifier 'all' in the definition of $\Delta \mathrm{F}$ escorts the expanding extension of the concept $\Delta C$ throughout its extensibility stages, so meaning differently along such levels. This ambiguity will turn out to play a fundamental role. Let's say that the universal quantifier is bounded by a concept/predicate $Q(x)$ when it heads a sentence of the form

$$
\forall x(\mathrm{Q}(\mathrm{x}) \rightarrow \phi)
$$

In that case, if $\mathrm{Q}$ is an extensible concept, the range of the quantifier can always be extended while it is still bounded by Q: the extensibility of the bounded quantifier's range follows the extensibility of the concept that bounds it.

In particular, the diagonal definition $\triangle D F$ is not yet available or given for quantification or reference when we proceed to define the enumeration out of which it diagonalizes; hence, it is not in the range of the quantifier by which we define $\Delta \mathrm{F}$.

It seems clear that in the definable universe, level of extensibility is the same as level of definability because, in the definable universe, an object becomes available or given exactly when it becomes definable. Thus at each new stage along the extensibility of the concept $\Delta \mathrm{C}$ we reach a new level of definability regarding sets of natural numbers: for each version of $\Delta \mathrm{F}$ and each definable enumeration $\triangle E F$ of it, the diagonal definition $\triangle \mathrm{DF}$ sits within a higher definability level than all the definitions in $\Delta \mathrm{F}$.

Even setting aside the paradoxes of definability and denotation, there are good reasons to believe that the concept of English language is extensible. Consider the following.

English, as any other natural language, is surely a function $f$ from syntactic objects to meanings (most probably an improper one-to-many function), that is to say, from signifiers to signifieds, as Saussure [I3] called them. Now there can be no English syntactic object $x$ such that $f(x)=f$, because no function can be defined in terms of itself. This means that we do not have in $f$ (i. e. in English) a name $x$ for $f$ (i. e. for English), that is to say, a name able to refer to the whole language in which it belongs. Since English $=\mathrm{f}$ and $\mathrm{f}$ ("English") $\neq \mathrm{f}$, English ("English”) $\neq$ English.

So when we speak in English about English, the 'English' in the metalanguage is not exactly the same as the 'English' in the object-language. And the best candidate to make part of the metalanguage but not of the object-language is precisely the expression 'English' as used in the metalanguage: when we speak in English about English, we may think we are determining a function $f$ by means of that same $f$, as in $f(x)=f$, but if what we are saying must be fully meaningful after all, circularity must in fact prevent us from referring to the whole $f$, so that in fact we are only referring to a previously determinate part $f^{*}$ of $f$. Then we do not have $f(x)=f$ but $f(x)=f^{*}$ where $\left\langle x, f^{*}\right\rangle$ is a member of $f$ but surely not a member of $f^{*}$.

Of course, any item in the metalanguage can be turned into a new item of an extended object-language and it is natural to suspect that this is what brings 
about extensibility.

It is a consequence of this approach that Cantor's theorem, when applied to $\mathrm{N}$ and interpreted in the full definable universe, has very little to do with cardinality, at least with cardinality in its strict sense, as in "a rectangle has more angles than a triangle." What Cantor's diagonal procedure reveals in this context is the extensibility of the concept of definite English definition of a set of natural numbers.

\section{IV}

It can be argued that the extensibility suggested by the Cantor-Richard case under consideration here is relative; the concept of definite English definition of a set of natural numbers is indefinitely extensible relative to the concept of countable set, so that for any characterization of its extension that yields a countable set $S$ there is one definition of the sort not in S. Shapiro and Wright [14] have formalized to a certain extent the notion of relative extensibility of concepts. We will offer here a weakened, simplified and set theoretic version (the authors' original characterization is typed and intensional; it is also stronger in a sense to be specified below).

First we define relative extensibility. A concept $\mathrm{CI}_{\mathrm{I}}$ is extensible relative to a concept $\mathrm{C} 2$ iff for every set $S$ falling under $\mathrm{C} 2$ of objects falling under $\mathrm{C}$ I there is an object $d$ such that

I. d falls under C I

2. $d \notin S$

The object $d$ is said to diagonalize out of $S$. For an example, take $C$ I as the concept of natural number and C2 as the concept of finite set; then the successor of the greatest number in $S$ will be a natural number not in $S$. Or take CI as the concept of real number and $\mathrm{C} 2$ as the concept of enumerable set. If we are right in this paper, the latter example is valid in the definable universe as well.

The idea is that there is no set falling under $\mathrm{C} 2$ that contains all the objects falling under CI and only them because for each set $S$ that is a C2 and contains only CI's there is a CI not in S.

We define now relative indefinite extensibility. A concept $\mathrm{CI}_{\mathrm{I}}$ is said to be indefinitely extensible relative to a concept $\mathrm{C} 2$ iff $\mathrm{CI}$ is extensible relative to $\mathrm{C} 2$ and the following holds:

3. $\{d\} \cup S$ falls under $\mathrm{C} 2$.

The idea is that there is no maximal set that falls under C2 and contains only Cis because for each set $S$ that falls under C2 and only contains Cis there is a new $C I$ that is not in $S$ and such that, added to $S$, gives a new set that also falls under C2. This is the type of extensibility roughly corresponding here to the 
Shapiro-Wright indefinite extensibility. The examples above are also examples of indefinite extensibility.

When $\mathrm{C} 2$ is the concept of set, so that there is no set at all of all the objects falling under CI, we will say that CI is absolutely extensible.

We said that this version is not only a simplified but also a weakened one because Shapiro and Wright require the existence of a function $f$ such that $d=f(S), f$ being the same through all extensibility stages; this may require strong formulations of the Choice Axiom.

According to this setting, we could just have that the concept of definite English definition of a set of natural numbers is indefinitely extensible relative to the concept of countable set. Now, it seems evident that the definitions up to some particular level of definability $\beta$ make up a countable set $S$ : after all, their signifiers can at most be countably infinite and at each given level $\beta$ each signifier should define just one set. In fact, each $S$ contains the extensibility of language only up to a certain level. This implies that the members of $S$ can be seen as members of one definite language $L$ in a hierarchy of languages, i. e. there is a possible human language $L$ such that $S \subseteq L$. Certainly, it appears that any humanly usable language must be countable. So $L$ must be countable and $\mathrm{S} \subseteq \mathrm{L}$; then $\mathrm{S}$ must be countable.

The claim that the available sets of natural numbers make up a countable set at any definability level implies that the extensibility here at stake is indefinite: we can go beyond any level we can define because we can diagonalize in the Richardian way out of any countable set of definitely definable sets of natural numbers. This should mean that the extensibility levels go up along all (definable) ordinals. This in turn implies that there is no set of all definitely definable sets of naturals and no set of all definite English definitions of sets of natural numbers. And this implies that sethood and enumerability disappear simultaneously in these cases. Indeed, this can be taken as an indication that in the definable universe all sets are countable. But this issue should be properly addressed as a part of a more general question concerning the set theoretic content of the full definable universe, a question well beyond our purpose here.

However a brief word about a possibly useful subset of standard mathematics may be appropriate here. As noted above, sets of natural numbers may be precisely defined within some language, L, containing all the usual logical equipment for Peano Arithmetic, augmented with the minimal mechanics for discussing sets of naturals. Thus the collection of 'explicitly definable (within L) sets of naturals' is itself well defined.

It is not, of course, itself definable within the language L, (thus avoiding Richard); indeed it is not even discussable there, being a higher-level collection than those definable within L. Nonetheless, it seems quite harmless, and possibly useful (particularly for real-analytic concepts such as lower upper bound), to be able to diagonalize out of it, as does Cantor.

For this purpose, it seems natural to extend the language L very slightly, to 
$\mathrm{L}_{1}$, which would allow one to speak precisely about formulas within L. Using this, we would have a much extended (though obviously still countable) collection of definable sets of naturals. Now we may continue in this Richardian manner to form increasingly extended languages $L_{2}, L_{3}, \ldots$ each being a similarly defined simple extension of the previous one. Though no technical details are presented here, the extension method is virtually identical in every case, so it does not seem to present any difficulty to pass to a supervening language $L_{\omega}$, that includes an isomorphic copy of each of the $L_{i}$. This process can then be started anew, exactly in the manner of ordinal number notations giving us $\mathrm{L}_{\omega+1}, \ldots, \mathrm{L}_{\omega+2}, \ldots, \mathrm{L}_{\epsilon_{0}}, \ldots$ and so on [II, Section II.7].

How far could such a procedure go? How far we can go defining Richardian diagonals depends on how far we can go defining ordinals. The latter depends in turn on the strictures we wish to impose on the notion of definability: if, for instance, we decide that an ordinal is only definable if there is a uniform device permitting to reach it from below, then, under the Church-Turing thesis, only the Church-Kleene constructive ordinals would exist in the definable universe and the first Church-Kleene non-constructive ordinal $\omega_{1}^{\mathrm{CK}}$ would set a natural limit on how far we can go on producing Richardian diagonals.

We will just add a consideration with the purpose of illustrating how indefinite extensibility should be expected to behave within the full definable universe. It is a fact that in the ordinal series we can easily define beyond whatever we can define. So, why can we not reach an uncountable ordinal by defining the set of all countable ordinals just as we do in classical set theory? Well, if all ordinals are countable, then surely the concept of 'countable ordinal' is as absolutely extensible as the concept of ordinal. So the scope of the bounded quantifier 'all countable ordinals' varies along the extensibility levels. For each level $\beta$ we can define an ordinal $\gamma$ as the set of all countable ordinals at $\beta$, and $\gamma$ will be a countable ordinal at $\beta+1$.

It is a corollary of Hartogs' theorem about cardinality that for any set $S$ there is an ordinal more numerous than $S$, but the proof of this theorem relies so essentially on the usual axioms of set theory (the powerset axiom, in particular), that it appears to be inapplicable in a definable universe.

In a sense, the powerset axiom bypasses both the issue of definability and the issue of extensibility by declaring, so to say, that all definitions and extensions have already been done. One is reminded of Russell's comment that postulation bears the same advantages over construction that theft does over honest toil.

It is time to address the crucial problem that any attempt to account for Cantor's theorem in the full definable universe has to cope with, namely: the multiplicity of definitely definable sets of naturals in the full definable universe cannot be countable (because of the Cantor-Richard proof), though it seems there is an at most countably infinite provision of the corresponding definitions. Let us call this the uncountability problem.

Cantor dealt with this problem in a way that strikes most of us as really 
unexpected of him. Cantor seems to have believed (at one time at least) that all the real numbers are definable, hence that the set of all definable real numbers must be uncountable. He wrote to Hilbert on August 8, 1906 [3. p. 446]

Wäre Königs Satz, daß alle "endlich definirbaren" reellen Zahlen einen Inbegriff von der Mächtigkeit $\aleph_{0}$ ausmachen, richtig, so hieß dies, das ganze Zahlencontinuum sei abzählbar, was doch sicherlich falsch ist. Es fragt sich nun, welcher Irrthum liegt dem angeblichen Beweise seines falschen Satzes zu Grunde? Der Irrthum (welcher sich auch in der Note eines Herrn Richard im letzten Hefte der Acta Mathematica findet, welche Note Herr Poincaré in dem letzten Hefte der Revue de Métaphysique et de Morale mit Emphase herausstreicht) ist, wie mir scheint, dieser: Es wird vorausgesetzt, dass das System $\{\mathrm{B}\}$ der Begriffe B, welche eventuell zur Definition von reellen Zahlenindividuen herangezogen werden müssen, ein endliches oder höchstens abzählbar unendliches sei. Diese Voraussetzung muß ein Irrthum sein, da sich sonst der falsche Satz ergeben würde: "das Zahlencontinuum hat die Mächtigkeit $\mathbf{N}_{0}$ ". Irre ich mich, oder habe ich Recht?

In our translation:

If König's statement that all "finitely definable" real numbers form a collection of cardinal number $\boldsymbol{x}_{0}$ were correct, this would imply that the whole continuum is countable, which is obviously wrong. The question is now on which error the purported proof of his false theorem is based. The error (which also appears in the note of a Mr. Richard in the last issue of the Acta Mathematica, which Mr. Poincaré emphasizes in the last issue of the Revue de Métaphysique et de Morale) is, it seems to me, the following: it is assumed that the System $\{B\}$ of notions $B$, which have eventually to be used for the definition of individual numbers, is finite or at most countably infinite. This assumption must be an error because otherwise this falsity would follow: "the numerical continuum has cardinality $\boldsymbol{\Sigma}_{0}$ ". Am I right or wrong?

It is usually believed that Cantor was wrong, that he failed to distinguish the set of the definable reals (which is usually deemed to be countable) from the set of the reals, most of which are (usually thought to be) indefinable. It seems very unlikely that Cantor failed to know that the possible syntactic pieces in any given language form an at most countably infinite set; still he refused to accept the set of notions eventually available for defining numbers to be countable.

But there is a way in which Cantor could be right. The multiplicity of definite English definitions of sets of naturals is not countable (and similarly the multiplicity of definitions of real numbers). The uncountability problem can be solved by adequately distinguishing between syntactical and semantical objects. To see how the supply of definitions can exceed the countable, consider that a definition is not a string of letters, not a mere syntactical object. An English definition is a pair 〈signifier, signified $\rangle$ in the function English, where 'signifier' and 'signified' are taken, as before, in the Saussurian sense: the signified is the syntactical object, i.e. the string of symbols, whereas the signified is 
the semantical object, i.e. roughly, Frege's Sinn. There is no doubt that the set $\Delta \mathrm{F}$ of all relevant signifiers is countable. If the function English mapped each signifier to only one signified, the set of all definite English definitions would be countable too. So the function English is for logical reasons an improper oneto-many function, at least for the signifiers at issue.

Since it is imposed on us by the definable version of Cantor's theorem together with elementary mathematical considerations, the ambiguity of such signifiers is not a matter of caprice or choice, not a matter of linguistic arbitrariness. It is just a necessity in need of explanation. But all the resources we need to develop that explanation have already become available in the course of our reflection. It is a fact that there are definite English definitions of sets of natural numbers that refer to 'all definite English definitions of sets of natural numbers'; for example, the diagonal definitions in the Cantor-Richard proof. And we know that this quantifiying phrase quantifies variously along the extensibility levels: it has a different range at each extensibility level. This provides the required source of ambiguity. Therefore, exactly the same insight that taught us how to deal with the Richardian diagonal enables us to solve the ensuing uncountability problem.

\section{V}

The ambiguity of quantification over definite English definitions of sets of naturals suggests a consideration about quantification and sethood.

The usual model theoretical thesis that any quantification domain is a non empty set and that any non empty set is a possible domain of quantification, must be adjusted in the definable universe in order to take account of the existence of definability levels. The best rationale for the model theoretical thesis mentioned above is the intuitive equivalence of the following propositions:

I. A multiplicity $M$ can be regarded as a completed totality

2. A multiplicity $M$ can be regarded as a single object

3. A multiplicity $M$ is a set

4. All the objects in a multiplicity $M$ are simultaneously available/given

5. It is possible to simultaneously quantify (make reference) over (to) all the objects in a multiplicity $M$

Consider that in a definable universe givenness or availability can be understood as definability. Since we are considering the existence of levels of definability, we must say that a multiplicity $M$ is a set if and only if there is a definability level $\beta$ such that all the objects in $M$ are definable at $\beta$.

The disadvantage of this general position is that it bans quantification over the extension of absolutely extensible concepts like the concept of set or the concept of ordinal. So it seems it would prohibit us from stating: 
[wF] No set is self-membered

or

[TR] All ordinals are transitive

A way out has been proposed by Luna [8] drawing on earlier suggestions by Gödel and Carnap. Propositions like [wF] and [TR] could be interpreted as expressing relations of entailment between concepts and properties:

[W $\left.\mathrm{F}^{\prime}\right]$ The concept of set entails the property of being non self-membered

[TR'] The concept of ordinal entails the property of transitivity

The quantifiers in [wF] and [TR] could be just apparent quantifiers if these propositions are taken to express relations of intensional entailment. As Gödel put it [7, p. 135, 136], 'all' need not mean in all cases the same as an infinite logical conjunction, it could mean "analyticity or necessity or demonstrability."

\section{APPENDIX}

Semantics for languages with absolutely extensible concepts: a sketch

Recall that an absolutely extensible concept is essentially one whose extension is neither empty nor a set. Standard semantics for formal languages, that is, standard model theory, deals only with domains of discourse that are sets. How would the semantics of a language including absolutely extensible concepts look?

A first issue is whether the semantics of natural language is itself adapted to extensible concepts. It may well be. When students first face the RussellZermelo paradox of the set $\mathrm{R}$ of all non self-membered sets, they feel there's some trick about it. Some of them even get to object something like 'I thought that the definition of $R$ meant all other sets, those previously given, not $R$ itself'. Those objections are usually suffocated by appeal to the rigid rules of instantiation of predicate logic.

Some years ago one of us asked his then I2-years-old daughter whether it is possible to put all boxes in the world into one box. She answered: 'yes, you just make a box large enough'. When her father objected that the large enough box could not contain itself, she replied: 'I meant all other boxes, of course'. Similarly, one can easily imagine Epimenides claiming he was referring to all other Cretans (of course!). So, one can argue that paradoxes are provoked by some unnatural semantics logicians and mathematicians impose on their artificial languages. It can be argued that natural languages are always used from a particular language level, which acts as a logical context determining the extension of the extensible concepts and the range of the quantifiers bounded by them. Then also meaning and truth conditions are determined by the logical context. Something on this line was rather timidly suggested by Parsons [9], 
then more resolutely recaptured by Glanzberg [5, 6]. If the role of context is so essential, there is little hope that a formalized theory of meaning and truth for languages with absolutely extensible concepts may be ever attained.

In any event, we can ask what general shape formal semantics should adopt in order to successfully deal with absolutely extensible concepts and avoid paradoxes. The core of semantics for formal languages is model theory. Indeed meaning and truth in formal languages is defined with respect to models. An interpretation relates a formal language to a particular model. What an interpretation provides is first of all a domain of discourse. In standard model theory the domain of discourse is fixed once for all. This trait is exactly what seems impossible to keep in any semantics suitable for a language with absolutely extensible concepts. A new kind of semantics allowing for extensible domains is required. We cannot develop here a full-fledged semantics with extensible domains; we shall just try to sketch where it deviates from standard semantics and how instantiation and generalization rules might be affected.

Let $C$ be an absolutely extensible concept and let $P(x)$ be the predicate ' $x$ falls under $C$ '. We call $P$ an absolutely extensible predicate. In dealing with absolutely extensible concepts the thing we must take care of is the range of the quantifiers (explicitly or implicitly) bounded by the corresponding absolutely extensible predicates. The quantifiers in subformulae like ' $\forall x(\mathrm{P}(\mathrm{x}) \rightarrow \cdots)$ ' and ' $\exists x(P(x) \& \cdots)$ ' can be said to be explicitly bounded by the absolutely extensible predicate P. But if we have chosen a set of Ps as our domain of discourse, then all quantifiers in our language are implicitly bounded by one of the variable extensions of $P$.

The point is then that the extension of $\mathrm{P}$, hence the range of the bounded quantifiers, can vary along the levels of language. This becomes especially relevant when we reach a new level precisely by using quantifiers bounded by absolutely extensible predicates; such are the diagonal objects $d$ mentioned on page 6: we quantify over a set of Ps in order to construct an object that diagonalizes out of that set while staying, nonetheless, within the extension of $P$. In such cases new objects, to which P applies, become available. The domain of discourse opened by $\mathrm{P}$ bas been extended.

Let us briefly consider an example taken from Luna [8]. Assume that the range of the quantifier and the variable in the following formula is some set of sets $S$ :

(I) $\forall x(x \in R \leftrightarrow x \notin x)$

In (I) we have a quantifier implicitly bounded by the predicate ' $x$ is in S' (and explicitly bounded by the predicate ' $x \notin x$ '). The formula defines a set $R$ which diagonalizes out of the whole range of the quantifier in (I); that is, $R$ diagonalizes out of the set of sets $S$ the formula quantifies over. As a consequence, $R$ is not in the range of the universal quantifier in (I): it lies on a higher extensibility level. Thus $R$ extends the domain of discourse. Of course, this precludes the classical inference to: 
(2) $R \in R \leftrightarrow R \notin R$

since $R$ is not in the range of $x$. From (I) we obtain by Existential Generalization:

$$
\text { (3) } \exists y \forall x(x \in y \leftrightarrow x \notin x)
$$

But, in so doing, we must provide the quantifier we introduce with an extended domain: generalization without domain extension should be forbidden in diagonalizing contexts. Consequently, we cannot get (2) from (3) because the range of the existential quantifier exceeds that of the universal quantifier: there is no guarantee that the $y$ is one of the $x$. Instantiation from a previous more restricted domain into a new and extended one is obviously invalid.

A model $M$ for a first order language $L$ is a pair $\langle D, f\rangle$ where $D$ is a domain of discourse (i. e. a non empty set) and $f$ is an interpretation function assigning members of $D$ to individual constants of $L$ and subsets of $D^{n}$ to $n$-ary predicates of $L$. In standard semantics we say that a sentence $P(d)$ in $L$, with $P$ a monadic predicate, is true in $M$ iff

$$
f(d) \in f(P) \subseteq D
$$

Now assume that the $d$ in $P(d)$ diagonalizes out of $f(P)$, as is the case in (I), while there is no other such diagonalization in the formula. What we need in order to provide truth conditions able to turn a formula like (I), which is unsatisfiable in standard semantics, into a satisfiable sentence is just shifting from a lower level language $L$ with a model $M=\langle D, f\rangle$ to a higher level language $L^{+}$ with an extended model $M^{+}=\left\langle D^{+}, f^{+}\right\rangle$such that $D \subset D^{+}, D^{+}-D \subseteq f^{+}(P)$ and $f^{+}(d) \in D^{+}-D$. We can reformulate this by considering an extensible language $L \cup L^{+}$with an extensible model $M M^{+}=\left\langle D, D^{+}, f, f^{+}\right\rangle$. Then we can say that a formula $\mathrm{P}(\mathrm{d})$ of $\mathrm{LUL}^{+}$is true in $\mathrm{MM}^{+}$iff at least one of the following conditions holds:

(i) $f(d) \in f(P) \subseteq D$

(ii) $f^{+}(d)$ diagonalizes out of $f(P)$ and $f^{+}(d) \in f^{+}(P) \subseteq D^{+}$and, for any other expression $\phi$ in $P(d), f^{+}(\phi)=f(\phi)$.

Now, in order to turn this rather vague characterization into a precise one, we would have to specify the conditions under which diagonalization (so understood and not, of course, in the Gödelian sense) occurs. There are many different ways in which one can diagonalize out of a set of Ps by means of a higher level P. Hence the precise formulation of extensible domain semantics will only be possible when a definite theory of diagonalization is available. Now the context dependence we have mentioned above and the fact that the hierarchy of extensibility levels goes beyond sethood cast serious doubts on the possibility of a complete formalization of truth conditions in this theory. 


\section{REFERENCES}

[I] BAAZ, M. 1996. "Infinite-valued Gödel logic with 0-1-projections and relativisations." Gödel'96: Logical Foundations of Mathematics, Computer Science, and Physics, volume 6 of Lecture Notes in Logic 23-33. PETR HÁJEK ed. Springer-Verlag, Brno.

[2] BOrel, E. 1908. "Les 'Paradoxes' de la Théorie des Ensembles." Annales Scientifiques de l'É.N.S., 3e série, tome 25, 443-448. Available at http://www . numdam.org/item?id=ASENS_1908_3_25_0

[3] Cantor, G. I99I. Briefe, H. mesch kows k i, w. Ni lson eds., Springer, Berlin.

[4] Dummetт, м. 1963. “The Philosophical Significance of Gödel's Theorem," in Truth and Other Enigmas, Duckworth, London, I978, I86-20I.

[5] Glanzbe Rg, M. 200I. "The Liar in Context." Philosophical Studies IO3, 217-25I.

[6] Glanzberg, M. 2004. "A Contextual-Hierarchical Approach to Truth and the Liar Paradox." Fournal of Philosophical Logic 33, 27-88.

[7] GÖDEL, K. 1944. "Russell's Mathematical Logic" in The Philosophy of Bertrand Russell, schi LPP ed. Northwestern University, Evanston, IL.

[8] Luna, L. 2008. "Can We Consistently Say That We Cannot Speak About Everything?" The Reasoner 2(9), 5-7.

[9] LUNA, L. 2009. "A Note on Formal Reasoning with Extensible Domains," The Reasoner 3(7), 5-6.

[Io] Parsons, C. 1974. "Sets and Classes." Nô̂s 8, I-I2. Reprinted in C. PARsons, Mathematics in Philosophy, Cornell University Press, Ithaca, I983, p. 209-220.

[II] POincaré, H. 1906. "Les Mathématiques et la Logique." Revue de Metaphysique et de Morale, I4, 294-317.

[12] ROGers, H. 1967. Theory of Recursive Functions and Effective Computability. McGraw-Hill.

[13] RUSSEll, B. 1906. "On Some Difficulties in the Theory of Transfinite Numbers and Order Types." Proceedings of the London Mathematical Society $4,29-53$.

[14] Saussure, F. I916. Cours de Linguistique Générale. Payot, 1972. 
[15] Shapi Ro, s. and wright, C. 2006. "All Things Indefinitely Extensible" in rayo, A. and uzQuiano, g. (eds.) Absolute Generality, Oxford University Press, 2006, p. 255-304. 
The Australasian fournal of Logic (ISSN I448-5052) disseminates articles that significantly advance the study of logic, in its mathematical, philosophical or computational guises. The scope of the journal includes all areas of logic, both pure and applied to topics in philosophy, mathematics, computation, linguistics and the other sciences.

Articles appearing in the journal have been carefully and critically refereed under the responsibility of members of the Editorial Board. Only papers judged to be both significant and excellent are accepted for publication.

The journal is freely available at the journal website at

$$
\text { http://www.philosophy.unimelb.edu.au/ajl/ }
$$

All issues of the journal are archived electronically at the journal website.

SUBSCRIPTIONS Individuals may subscribe to the journal by sending an email, including a full name, an institutional affiliation and an email address to the managing editor atajl-editors@unimelb.edu.au Subscribers will receive email abstracts of accepted papers to an address of their choice. For institutional subscription, please email the managing editor at ajl-editors@unimelb.edu.au

Complete published papers may be downloaded at the journal's website at http://www.philosophy.unimelb.edu.au/ajl/ The journal currently publishes in pdf format.

Submission The journal accepts submissions of papers electronically. To submit an article for publication, send the ${ }^{\Delta} \mathrm{T}_{\mathrm{E}} \mathrm{X}$ source of a submission to a member of the editorial board. For a current list of the editorial board, consult the website.

The copyright of each article remains with the author or authors of that article. 